\title{
Principales características e implicaciones del marketing online.
}

Main characteristics and implications of online marketing.

Miguel Ángel Sánchez Jiménez. ${ }^{1}$, María Teresa Fernández Alles. ${ }^{2}$ \& Juan José Mier-Terán Franco. ${ }^{3}$

Recibido: 05-06-2017 / Revisado: 06-08-2017 Aceptado: 12-09-2018/ Publicado: 01-10-2018

\begin{abstract}
.
DOI: https://doi.org/10.33262/cienciadigital.v2i4.211

Nowadays, new technologies have forced companies to modify their marketing strategies to adapt them to a new way of acting in relation to the market. A large majority of companies have developed tools and adapted their activities to the different media, resources and environments that have emerged over time, such as the Internet as a marketing tool. In this way, this study proposes, through a bibliographic review, to know the main characteristics that online marketing generates in organizations, as well as the main implications that have arisen in this matter such as online marketing from the business perspective, online advertising and relationship marketing and customer loyalty through the Internet.
\end{abstract}

Keywords: Online Marketing, Social Media Marketing, Business Strategy, Loyalty.

\section{Resumen.}

En la actualidad las nuevas tecnologías han obligado a las empresas a modificar sus estrategias de marketing para adaptarlas a una nueva forma de actuar respecto al mercado. Una gran mayoría de las empresas han desarrollado herramientas y adaptado

\footnotetext{
${ }^{1}$ Universidad de Cádiz, Departamento de Marketing y Comunicación, Cádiz, España, miguelangel.sanchez@uca.es

${ }^{2}$ Universidad de Cádiz, Departamento de Marketing y Comunicación, Cádiz, España, teresa.alles@uca.es

${ }^{3}$ Universidad de Cádiz, Departamento de Marketing y Comunicación, Cádiz, España, juanjose.mier-teran@uca.es
} 
su actividad a los diferentes soportes, recursos y entornos que han ido surgiendo a lo largo del tiempo, tal es el caso de internet como instrumento de marketing. De esta manera en este estudio se plantea, a través de una revisión bibliográfica, conocer las principales características que el marketing online genera en las organizaciones, así como de las principales implicaciones que han surgido en esta materia como son el marketing online bajo la óptica empresarial, la publicidad online y el marketing relacional y la fidelización de clientes a través de internet.

Palabras Claves: Marketing Online, Social Media Marketing, Estrategia Empresarial, Fidelización.

\section{Introducción.}

Internet ha supuesto sin duda la mayor revolución acaecida en estos últimos 20 años, no sólo en el ámbito social sino también comercial, posibilitando un acceso rápido y sencillo a una gran cantidad de información, permitiendo, asimismo, una comunicación directa con cualquier lugar del mundo. Desde su creación, a principios de los años 90, internet ha experimentado un crecimiento masivo y continuo que aún sigue dándose en la actualidad. En 2017 más de la mitad de la población mundial será usuaria y tendrá acceso a internet, llegando a alcanzar más de un $70 \%$ de penetración en Oceanía, Europa y Norteamérica (Internet World Stats, 2017). En 2017 hay contabilizadas más 128 millones de páginas web con dominio comercial, es decir, compañías que generan parte de su actividad económica en internet (Dailychanges, 2017).

Cuando empezó a percibirse internet como una herramienta importante que podía afectar a la estrategia de marketing, muchas empresas la tuvieron en cuenta en su actividad empresarial (Celaya, 2011). A lo largo de los años la red se ha ido convirtiendo en un elemento esencial en las empresas para mantener una relación eficiente con sus clientes, por lo que el marketing en internet cobra una gran relevancia en las estrategias empresariales (Balmer y Yen, 2017).

El desarrollo de estrategias de marketing en internet, también conocido como marketing digital o marketing online (denominación esta última que se utilizará de aquí en adelante) se define como "la comercialización y comunicación de productos y servicios a través de las posibilidades que ofrece internet" (Millán, 2008). El marketing online es un término amplio que integra acciones, técnicas y herramientas que se desarrollan usando internet.

En relación a este concepto surgen diversos enfoques que tratan de aprovechar las nuevas tecnologías entre los que vamos a destacar los referidos al social media marketing y al marketing móvil.

De esta manera, el objetivo de este estudio es conocer las principales características e implicaciones que el marketing online genera en las empresas, así como de los principales 
enfoques que han surgido con las nuevas tecnologías como son el social media marketing y el marketing móvil.

\section{Marketing online: concepto y características.}

Durante las últimas décadas, el marketing ha experimentado un considerable desarrollo, paralelo a la propia evolución de las sociedades en las que se extiende su ámbito de actuación. Se conciben nuevas formas de entender los mercados y a los consumidores, se utilizan nuevos instrumentos y tecnologías para obtener información, se buscan nuevos métodos para desarrollar las relaciones de intercambio con los consumidores y se potencian nuevas formas de comunicación con ellos (Mier-Terán Franco, 2004).

Como consecuencia de la aparición de internet surge en la ciencia del marketing una rama específica denominada marketing en internet o marketing online que consiste en la utilización de internet con finalidades de marketing. El marketing online se define como "la comercialización y comunicación de productos y servicios a través de las posibilidades que ofrece internet" (Millán, 2008). Liberos (2013) considera internet una valiosa herramienta de marketing con la que comunicar y distribuir los productos, ya sean bienes, servicios o ideas. Además, internet es también un recurso para la investigación comercial y de mercados con el que se obtiene información sobre los hábitos de los consumidores finales, las actuaciones de los competidores, etc. Por otra parte, el marketing online ha originado nuevas necesidades, conceptos, formatos y, en consecuencia, nuevas estrategias. El reto en las empresas consiste en aprovechar todas las ventajas que ofrece el marketing en internet para incluirlo en su planificación estratégica, teniendo como objetivo principal convertir a los usuarios de internet en clientes potenciales (Strauss, 2016).

Según Mayordomo (2004) las características fundamentales del marketing online son las siguientes:

- El marketing online es un sistema de pensamiento, una filosofía que engloba tanto al propio marketing como a las técnicas que utiliza.

- El marketing online representa una ventaja competitiva, especialmente para las pequeñas empresas.

- Los objetivos y conceptos del marketing online y del marketing tradicional no cambian.

- Debe formar parte de la cultura general de la empresa.

- Permite la comercialización de productos y servicios a través de internet.

- Es un gran igualador de oportunidades entre las empresas grandes y pequeñas.

- Con el marketing online el público ya no es el receptor pasivo del mensaje, sino que será este quien busque el producto que más le interesa y cubra sus necesidades.

- Internet es en sí mismo un catálogo permanentemente actualizado.

- Con el marketing online se produce un cambio importante, en el que se pasa de un 
marketing basado en el producto a otro centrado en el cliente.

Una vez especificada la definición y los aspectos principales del marketing online, conviene destacar cuáles son las cuatro variables mix del marketing online. En este sentido, Merodio (2010) estima que internet es uno más de los instrumentos de los que dispone el marketing en la empresa para conseguir sus objetivos y metas. De ahí que sea necesario integrar adecuadamente la estrategia para internet en las estrategias generales de marketing de la organización. Los programas de marketing en internet difieren de los programas en los entornos convencionales como consecuencia de dos rasgos principales de internet: su naturaleza abierta y global, y su elevado grado de interactividad. Considera que de las 4 variables del marketing mix tradicional o 4 Ps: Producto, Precio, Distribución y Comunicación, se pasa a las 4 Cs del marketing online:

- Cliente: considera que en el marketing online el cliente es el centro de todo y tiene poder absoluto, en el que las empresas tendrán que diseñar productos y servicios a medidas para satisfacer realmente sus necesidades.

- Comunicación interactiva: se basa en el feedback o retroalimentación en el cual la empresa debe invitar a los usuarios que sean uno más, compartiendo sus experiencias con otros usuarios y con la propia empresa.

- Coste: a los clientes les acarrearía menor coste el adquirir un producto o servicio en internet, tanto por precio, desplazamientos, esfuerzos, etc.

- Conveniencia: consiste en llegar al cliente mejor y de forma más rápida.

\section{Implicaciones del marketing online.}

En este apartado se hace referencia a las investigaciones más relevantes llevadas a cabo sobre el marketing online. Cabe resaltar la multitud de estudios existentes en materia de marketing online, siendo fundamentalmente cuatro los campos en los que quedarían enmarcados: el marketing online bajo la óptica empresarial, la publicidad online y el marketing relacional junto con la fidelización de clientes a través de internet.

\subsection{Marketing online bajo la óptica empresarial.}

En este campo, las primeras investigaciones surgieron a mediados de los años 90 cuando se vislumbraba la gran importancia que tendría la red informática de manera global tanto para la sociedad como para el negocio empresarial. Estos primeros estudios se refieren a la repercusión que tiene y podría tener el marketing en internet en el comercio, así como las oportunidades y amenazas con las que se encuentran las empresas ante este grandísimo cambio que está emergiendo cada vez con más fuerza en el entorno. Las primeras investigaciones estudiaron el potencial de integración de internet en el entorno empresarial, en el uso del correo electrónico, las bases de datos, las fotografías, los videos y la obtención de información (Sterne, 1995; Peterson, 1997; Bennet, 1998). Además, se considera que la principal preocupación en las empresas era el uso del marketing en internet para aumentar 
las ventas y conseguir así mayores beneficios (Granger y Schroeder, 1994).

Mehta y Sivadas (1995) analizaron las actitudes de los consumidores ante la comunicación en la red y establecieron que internet permite a las empresas dirigir y comunicarse con los consumidores involucrados e interesados. Además, por primera vez se destaca la importancia de la utilización del correo electrónico en la empresa, donde cualquier mensaje puede ser fácilmente enviado a cualquier parte remota del mundo en un tiempo muy corto. En este mismo sentido, Kambil (1995) señala la importancia de tener en cuenta el comercio electrónico en la empresa, mejorando así la innovación, la producción, las ventas y los procesos, consiguiendo menos costes y un aumento de la productividad. Además, este autor es el primero en destacar la importancia de la integración de internet en la estrategia de la empresa y las nuevas oportunidades y amenazas, en la que la adopción generalizada del comercio electrónico incrementará su productividad al hacer transacciones sustancialmente más eficientes.

En los años posteriores comienzan a surgir nuevos cambios en el entorno que afectan al marketing online, como el uso de nuevas herramientas (los sitios web, los buscadores, el correo electrónico, las noticias, etc.) que permitiría a las empresas atender mejor a sus clientes y añadir valor a sus productos (Heinen, 1996). Además, la globalización comenzó a desarrollarse con mucha fuerza a escala mundial, interrelacionando cada vez más los mercados y afectando al marketing online (Quelch, 1996).

Ante esto, el marketing online se presenta como una potente arma en la apertura de la empresa a los mercados internacionales. Hamill (1997) investigó el profundo impacto de internet en el marketing internacional, examinando la repercusión en los intermediarios, el impacto en los mercados globales, las alianzas estratégicas, la fijación de precios internacionales, la publicidad global y especialmente la facilidad de acceso de las pequeñas y medianas empresas a los mercados globales. Teniendo en cuenta esta mayor preocupación de las empresas a abrirse a otros mercados, se realizan estudios sobre las funciones de internet como canal de distribución. Así, en la investigación realizada por Peterson et al. (1997) se analiza las similitudes y diferencias entre un canal de distribución tradicional y uno online, y qué productos y servicios son más adecuados para cada tipo de distribución, proporcionando un marco teórico acerca de los posibles impactos de internet en la comercialización de los productos y servicios a los consumidores. Así, consideran internet como un canal de distribución importante para algunos productos y servicios (viajes, libros, servicios bancarios, asesoramiento, etc.) que pueden ser vendidos e incluso enviados de forma electrónica (libros electrónicos, software, archivos, etc.).

La importancia del marketing online ha ido aumentando con el paso de los años, surgiendo muchas empresas que comercializan sus productos y servicios a través de internet. De esta manera comienzan a desarrollarse estudios más específicos. Breitenbach y Van Doren 
(1998), realizaron un análisis cuantitativo a más de 50 páginas web de empresas, identificando qué técnicas de marketing en internet llevan a cabo y su manera de llegar a los usuarios para alcanzar los objetivos de marketing. En este estudio se determina que el desafío de las empresas en el empleo del marketing online es proporcionar a los usuarios una experiencia que no se puede conseguir fácilmente en los medios de comunicación tradicionales, además de las enormes posibilidades que ofrece internet en la variedad y personalización de los productos a los consumidores. Las páginas web deben proporcionar un valor adicional a los usuarios, deben dar una razón para que estos lo visiten y vuelvan a repetir la visita.

Por otro lado, cada vez hay menos interés por parte de las empresas de dirigir su misma oferta comercial a un mercado masivo y empieza a cobrar importancia la segmentación. En este sentido, Wu (2002) estudió el comportamiento del consumidor en el marketing online. Señala que los consumidores se dividen en varios grupos o segmentos teniendo en cuenta diversos factores como el estilo de vida, las características personales, etc. Estos factores afectan a los consumidores en el marketing online y a su grado de implicación en el comportamiento de decisión de compra, los artículos comprados, la cantidad de dinero empleado y el volumen de compra. De esta manera la empresa debe establecer una estrategia de marketing idónea para cada grupo de consumidores, estableciendo un marketing cada vez más personalizado. En esta misma línea Tian (2002), teniendo en cuenta el alto grado de internacionalización y apertura de nuevos mercados que lleva consigo los negocios a través de internet, hizo referencia a las diferencias culturales y la implementación de un marketing online teniendo en cuenta la cultura a la cual se dirige para vencer a la competencia global.

No sólo es necesario una segmentación del mercado, sino también proporcionar un producto o servicio de calidad. Parasuraman (2002) realizó un estudio sobre la importancia en la prestación de servicios de calidad a través de las páginas web como estrategia de marketing, siendo estas esenciales para el éxito, por encima de otras estrategias como las de bajo precio. Estudia como los clientes perciben y evalúan un buen servicio de calidad en la red para que los directivos de las empresas puedan aplicarlo eficientemente. Además, analiza las diferencias entre un servicio de calidad en negocios tradicionales con los realizados a través de internet.

Para Fernández (2016), el marketing online o digital sigue los mismos principios que el marketing tradicional aplicado a un entorno virtual. A través de los sitios web, comercios electrónicos o anuncios por internet, las organizaciones canalizan su túnel de ventas a través de los medios digitales, tratando de satisfacer y despertar o generar deseos de compra en el consumidor, manteniendo esa satisfacción a través del tiempo.

Una de las principales ventajas que proporciona el marketing en internet para las empresas es el mayor conocimiento de los usuarios mediante la investigación de mercados. Maity y 
Okleshen Peters (2005) realizaron un estudio sobre la investigación comercial en internet. Exponen que estas investigaciones pretenden analizar la experiencia del consumidor online tanto en términos de cantidad y calidad en sus visitas a una página web. Este artículo revisa qué técnicas se usan en la investigación de mercado online. Se especifica que las técnicas o métodos más tradicionales deben combinarse con otros más recientes, como los referidos a la usabilidad, para conseguir una evaluación más completa de la actividad de los consumidores online. La usabilidad en internet se refiere a la capacidad de usar una página web fácilmente y de manera intuitiva. Una página web es usable cuando los usuarios encuentran rápidamente lo que buscan y pueden navegar de manera fácil e intuitiva para encontrar información valiosa.

Para las empresas, las reglas de juego del mercado cambian rápidamente y el aspecto digital lo está cambiando todo (Leeflang, 2014). No sólo se trata de la tecnología, sino también de los cambios en la actitud y en el comportamiento de los consumidores, quienes se motivan por el uso de las nuevas tecnologías. Así mismo, el universo digital se extiende en la sociedad y genera nuevos estilos de vida y modernos hábitos de consumo.

\subsection{Publicidad online.}

En segundo lugar, se hace mención a otra de las grandes áreas de estudio del marketing online, la publicidad online. Las primeras formas publicitarias del marketing online son una traslación de la publicidad de medios tradicionales a internet, además de los textos y enlaces en la página web toma especial relevancia los denominados "banner", un hecho documentado por primera vez por Briggs y Hollis (1997), quienes definen el banner como un anuncio rectangular o cuadrado que puede ser estático o dinámico, diseñado con herramientas multimedia a los cuales se les pueden aplicar imágenes en movimiento, animación o audio. El banner está vinculado a la página web del anunciante, ya que si se pulsa transporta el navegador a dicha página web. Puede colocarse en cualquier parte de la página web y puede tener cualquier tamaño y estilo. Además, como ventaja consideran que puede monitorear (supervisar) en tiempo real y que va dirigido a los intereses específicos de los navegantes. Otro tipo de publicidad online que surge son los denominados "pop-up" (Hoffman y Novak, 1997), que consisten en ventanas emergentes de tamaño variable que se abren de manera automática y flotante en la pantalla cuando se carga una página o se pulsa un enlace. Consideran que, aunque a veces es más efectivo que el banner, puede ser que algunos usuarios de internet lo consideren intrusivo o molesto.

Los cambios que experimenta internet a comienzos del siglo XXI tienen repercusiones en los nuevos estudios de publicidad online que se realizan en los próximos años.

En este sentido, Spencer y Giles (2001) escriben acerca del gran cambio que se está dando en el marketing online con la revolución de internet y la forma en que los consumidores interpretan la publicidad. Comenta que en el futuro los consumidores rechazarán la 
publicidad estática, concretamente los banners, y abre la puerta a que surjan nuevas herramientas de comunicación más eficaces. De esta manera surgen nuevas formas publicitarias, Armstrong (2002) se mostró crítico con la forma tradicional del banner y resalta la importancia del patrocinio como nueva forma mejorada de publicidad online. Mediante el patrocinio una empresa presenta una marca o producto en la página web de otra empresa. De esta forma se presenta el producto del patrocinador en diferentes formatos (texto, foto, video, etc.) en una página web cuyo contenido esté relacionado con la del propio producto. Este tipo de publicidad online tiene como ventajas la segmentación del público objetivo (por los temas y los contenidos tratados en cada web) y la escasa inversión necesaria. Otra forma de publicidad online que surge con fuerza es la publicidad contextual. En el estudio de Muela et al. (2005) la publicidad contextual se define como la publicidad segmentada según las preferencias o intereses del usuario, donde un sitio web inserta anuncios basados en textos, gráficos o videos, y recibe una cantidad de dinero por cada clic que el visitante haga sobre el anuncio. Por lo tanto, es una herramienta del marketing online en la que una página web muestra publicidad de productos de otra empresa en las que el usuario se ha mostrado interesado previamente. El ejemplo más usado de publicidad contextual pertenece a la herramienta Adsense de Google.

Un aspecto relevante sobre la publicidad online es el marketing en motores de búsqueda o search engine marketing (SEM). El SEM es una forma de marketing en internet que busca publicitar páginas web mediante el aumento de su visibilidad en los motores de búsqueda habilitados en algunas páginas (Google, Yahoo, Bing, etc.) (Millán, 2008). Respecto a las investigaciones más significativas cabe destacar la aportación de Sen (2005), el cual establece que el SEM engloba dos métodos para realizar publicidad de una página web a través de buscadores. Primero la búsqueda de pago, que pretende aumentar el tráfico de la página web a través de la compra de anuncio en los buscadores. A veces también se le conoce cómo Pay Per Click (PPC) ya que en la mayoría de estos anuncios el importe a pagar varía según los clicks que los usuarios realicen en el anuncio. El segundo es conocido como optimización de motores de búsqueda o search engine optimization (SEO), que consiste en el proceso de mejorar la visibilidad de la página web en los buscadores de manera natural, sin realizar un pago. Los motores de búsqueda usan los rastreadores para recoger información utilizando criterios predefinidos, así, el contenido recogido se clasifica e indexa para la búsqueda rápida y el acceso. Los motores de búsqueda son ampliamente utilizados por los usuarios de internet para localizar el contenido relevante de acuerdo a las palabras clave presentadas (Zineddine, 2016). Tiene como objetivo aparecer en las primeras posiciones de los buscadores y aumentar el tráfico de visitas de una página web. El estudio de Wilson y Pettijohn (2008) se centra en los fundamentos de la optimización de motores de búsqueda (SEO), analizando si es mejor para la empresa vendedora externalizar este proceso, dejándolo en manos de otra empresa más experta, debido a la dificultad y el desconocimiento que tienen algunas empresas vendedoras en conseguir buenos resultados a través de SEO. Por último, en el estudio de Iglesias-García y Codina (2016) resaltan la importancia estratégica del SEO, en el que los 
algoritmos de los motores de búsqueda, cada vez más complejos, tienden a centrarse en la experiencia del usuario y en destacar aquellos contenidos de mayor calidad. Así, se habla de un nuevo SEO, que ha pasado a denominarse, en lugar Search Engine Optimization, como Search Experience Optimization, porque se centra en optimizar la experiencia de los usuarios, no en la del buscador.

Con la aparición de las redes sociales surgió el concepto publicidad 2.0 referida a los anuncios publicitarios a través de dichas plataformas. Moreno (2014) especifica que las redes sociales han encontrado en la publicidad la vía para obtener ingresos económicos. Algunas de ellas como Facebook, Twitter o Linkedln han conseguido grandes beneficios gracias a la comercialización de espacios publicitarios. Entre los motivos de la proliferación de este tipo de publicidad se encuentra la mayor interacción que generan los anuncios y la alta posibilidad de segmentación de los usuarios. Muchas empresas se deciden a intervenir en las redes sociales por la posibilidad que ofrecen de llegar a su público objetivo fácilmente, definiendo claramente a quien quiere que llegue su publicidad, cosa que no se podría realizar con tanta eficiencia con los espacios publicitarios más tradicionales. En el estudio de Boateng y Okoe (2015) destacan que, en general, los usuarios tienen una actitud positiva hacia la publicidad en los medios sociales y lo consideran como un buen canal de comunicación. Sin embargo, estas respuestas de comportamiento dependen tanto del uso de publicidad en medios sociales, como de la reputación corporativa. Por lo tanto, las empresas que tienen la intención de utilizar la publicidad en los medios sociales deben construir una imagen corporativa y deben identificarse con sus clientes para generar confianza.

\subsection{Marketing relacional y fidelización de clientes a través de internet.}

Otro campo objeto de estudio sobre la materia que se está analizando es el referido al marketing relacional y fidelización de clientes a través de internet, en el que se ofrece un servicio cada vez más orientado al consumidor. Comienzan a aparecer estudios enfocados a la gestión de las relaciones con los clientes (CRM) y el marketing relacional a través de internet. O'Leary y Perry (2004) estudian el CRM como modelo para recoger información de los clientes en internet y obtener una base de datos que mejore la gestión de las relaciones con los clientes. Los resultados mostraron que la obtención de esta base de datos a través de internet ofrece ventajas para la empresa, tales como: velocidad en la obtención de datos, datos más precisos y un ahorro en los costes que repercutirá en unas mejores relaciones con los clientes. También se destaca la importancia en la colaboración entre los equipos de marketing y los de tecnología de la información para conseguir el éxito en la obtención de datos online. En la publicación de Newell (2001) amplía el concepto de CRM en el marketing online, el cual no se basa sólo en la integración entre internet y la base de datos, sino que lo considera un modelo de gestión de toda la organización centrada en la orientación al cliente. Este enfoque está estrechamente vinculado con el marketing relacional y el marketing personalizado, tratando de aplicar los estudios realizados por Peppers (1993) sobre marketing relacional y personalizado al contexto de internet. Newell 
(2001) expone que la empresa será más eficiente en su negocio online en la medida que sea capaz de mantener relaciones duraderas y a largo plazo con sus clientes, consiguiendo que sean fieles o leales a la empresa. Esto se consigue mediante una estrategia de personalización que consta de cuatro pasos:

1. Primero se debe identificar cuáles son los clientes o futuros clientes de la empresa que formarán parte de su base de datos.

2. En segundo lugar, se debe añadir información para diferenciar los clientes por sus características o necesidades particulares.

3. Posteriormente la empresa debe interactuar con los clientes y realizar un seguimiento individual para aumentar el conocimiento de cada uno.

4. Por último, la empresa debe personalizar o tratar a cada cliente de manera distinta, diseñando un producto o servicio para cada uno de ellos.

Continúa la preocupación del marketing online por la fidelización de los clientes y por el servicio orientado al consumidor. Taylor y England (2006) investigaron sobre las posibilidades que ofrece el diseño de las páginas web en el marketing online, destacando la importancia de agrupar correctamente los contenidos de manera que los haga más accesible al usuario. Desde la perspectiva del marketing, esta mejora en el acceso a los contenidos aumenta la probabilidad de que los productos y servicios ofertados en la página web sean adquiridos por el consumidor. Para que los consumidores utilicen una página web y la vuelvan a utilizar en el futuro, tienen que encontrar lo que necesitan de manera sencilla.

Salazar et al. (2016) destacan como uno de los principales principios del marketing online conseguir una mayor fidelización de los clientes. Considera que el fin del marketing online es estar en contacto constante con los clientes y puedan consultar en cualquier momento los productos y/o servicios, generando confianza y seguridad. Con una buena estrategia de comunicación en una página web el cliente podría acceder a diferentes fuentes de información, así como dejar su opinión, sus dudas, sus quejas, etc., respetando todas las opiniones y respondiendo en todos los casos. Esto dará buena imagen cuando nuevos clientes accedan al sitio, fidelizándolos de una forma sencilla y atractiva y manteniéndolos interesados en la empresa sobre el resto de las competidores. Asimismo, la fidelización del cliente es necesario hoy día debido a la creciente competencia, por lo que la empresa debe intentar conservar a sus clientes, procurando la satisfacción plena de éstos con los productos, generando un vínculo emocional.

\section{Conclusiones.}

- Puede observarse mediante la revisión de la literatura las grandes implicaciones que ha tenido y tiene actualmente el marketing a través de internet y los medios digitales. Paralelamente al crecimiento y la popularización de internet en todo el mundo, surgió su aprovechamiento por las empresas permitiendo el acceso a nuevas herramientas y 
a un gran mercado al cual dirigir su negocio. Internet ha evolucionado a lo largo del tiempo, el principal gran cambio experimentado en la red fue a partir del año 2006 con el paso de la web 1.0 o estática, en la que el contenido era controlado por las organizaciones, a una web 2.0 o social, que consideraba al usuario como parte central de la red, pasando a una actitud más colaborativa, en el que la principal prioridad para las empresas no es vender lo máximo posible, sino escuchar y participar en la conversación que se da en internet.

- Para aprovechar las posibilidades que ofrece internet en el ámbito comercial aparece el marketing online, que se define como la comercialización y comunicación de productos y servicios a través de internet. El marketing online proporciona diferentes ventajas en el ámbito empresarial entre las que destacan: el acceso a nuevas herramientas de marketing, la apertura a mercados internacionales, la mejora de la experiencia del usuario, la mayor facilidad para realizar la segmentación, un mayor conocimiento de los usuarios mediante la investigación de mercados, etc.

- Respecto a la publicidad online las empresas disponen de internet como nuevo canal de comunicación, desarrollando nuevas formas publicitarias que suponen un cambio radical respecto a la publicidad tradicional. Además, el marketing online también permite relacionarse con el consumidor de forma más sencilla y atractiva, generando un vínculo emocional y favoreciendo así la fidelización del cliente.

- Así, este estudio proporciona una base sólida para conocer mejor la importancia marketing digital y desarrollar estrategias a través de los dispositivos móviles y las redes sociales por parte de las organizaciones.

\section{Referencias bibliográficas.}

Armstrong, S. (2002). "La publicidad en internet". Ediciones Deusto. Bilbao.

Balmer, J. M., y Yen, D. A. (2017). "The Internet of total corporate communications, quaternary corporate communications and the corporate marketing Internet revolution". Journal of Marketing Management, 33(1-2), 131-144.

Bennett, R. (1998). "Using the World Wide Web for international marketing: Internet use and perceptions of export barriers among German and British businesses". Journal of Marketing Communications, 4(1), 27-43.

Boateng, H. y Okoe, A. F. (2015). "Consumer's attitude towards social media advertising and their behavioral response". Journal of Research in Interactive Marketing, 9(4), 299312

Breitenbach, C. S. y Van Doren, D.C. (1998). "Value-added marketing in the digital domain: 
Enhancing the utility of the Internet”. The Journal of Consumer Marketing, 15(6), 558575.

Briggs, R. y Hollis, N. (1997). "Advertising on the web: Is there response before clickthrough?”. Journal of Advertising research, 37(2), 33-46.

Celaya, J. (2011). La empresa en la web 2.0. Gestión 2000. Grupo Planeta. Barcelona.

Chris O'Leary, S. R. y Perry, C. (2004). "Improving customer relationship management through database/Internet marketing: A theory-building action research project". European Journal of Marketing, 38(3), 338-354.

Dailychanges. (2017). "DailyChanges.com: Daily DNS Changes and web Hosting Activity". Recuperado el 27 de marzo de 2017 en: http://www.dailvchanges.com/

Fernández, P. V. (2016). Metodología para la elaboración de un plan de marketing online. 3C Empresa, 5(2), 57.

Granger, M. J. y Schroeder, D. L. (1994). "Integrating the Internet into the business environment”. Industrial Management + Data Systems, 94(8), 37-37.

Hamill, J. (1997). "The Internet and international marketing". International Marketing Review, 14(5), 300-323.

Heinen, J. (1996). "Internet marketing practices". Information Management \& Computer Security, 4(5), 7-14.

Hoffman, D. L. y Novak, T. P. (1997). "Measuring the flow experience among web users". Interval research corporation, 31.

Iglesias-García, M. y Codina, L. (2016). "Los cibermedios y la importancia estratégica del posicionamiento en buscadores (SEO)”. Opción, 32(9).

Internet World Stats (2017). "World internet Users and Population Stats". Recuperado el 2 de abril de 2017 en: http://www.internetworldstats.com/stats.htm

Kambil, A. (1995). "Electronic commerce: Implications of the Internet for business practice and strategy". Business Economics, 30(4), 27-27.

Leeflang, P. S., Verhoef, P. C., Dahlström, P. y Freundt, T. (2014). "Challenges and solutions for marketing in a digital era". European management journal, 32(1), 1-12.

Liberos, E. (2013). El libro del marketing interactivo y la publicidad digital. ESIC Editorial. 
Maity, M. y Cara Lee, O. P. (2005). "A primer for the use of Internet marketing research tools: The value of usability studies". Interactive Marketing, 6(3), 232-245.

Mayordomo, J. L. (2002). E-marketing. Gestión 2000.

Mehta, R. y Sivadas, E. (1995). "Direct marketing on the Internet: An empirical assessment of consumer attitudes”. Journal of Interactive Marketing, 9(3), 21-21.

Merodio, J. (2010). Marketing en Redes Sociales: Mensajes de empresa para gente selectiva. Juan Merodio.

Mier-Terán Franco, J. J. (2004). "Marketing socioambiental: Un nuevo paso en el desarrollo del marketing social”. International Review on Public and Nonprofit Marketing, 1(1), 139-153.

Millán Tejedor, R.J. (2008). Marketing online. Creaciones Copyright. España.

Moreno, M. (2014). El gran libro del Community Manager. Gestión. Barcelona.

Muela, C.; Sánchez Cid, y Baraybar, A. (2005). "Publicidad contextual una alternativa de la eficacia en Internet". Area abierta. No. 12. 2005.

Newell, F. (2001). Loyalty. com: Customer relationship management in the new era of Internet marketing. McGraw-Hill, Inc.

Parasuraman, A., Zeithaml, V. A. y Malhotra, A. (2002). "Service quality delivery through web sites: A critical review of extant knowledge". Academy of Marketing Science.Journal, 30(4), 362-362.

Peppers, D. (1993). The one to one future: building relationship one customer at a time.

Peterson, R. A. (1997). Electronic marketing and the consumer. Sage Publications, Inc.

Peterson, R. A., Balasubramanian, S. y Bronnenberg, B. J. (1997). "Exploring the implications of the Internet for consumer marketing". Academy of Marketing Science.Journal, 25(4), 329-346.

Quelch (1996). "The Internet and International Marketing". Sloan management review, $37(3), 60-75$.

Salazar, L. J. C., Matta, J. R. T., Llatas, F. D. H. y Calderón, R. F. C. (2016). "Marketing online y la fidelización del cliente en una PYME de autopartes-chiclayo". tzhoecoen, $8(2)$. 
Sen, R. (2005). "Optimal search engine marketing strategy". International Journal of Electronic Commerce, 10(1), 9-25.

Spencer, C. y Giles, N. (2001). "The planning, implementation and evaluation of an online marketing campaign”. Journal of Communication Management, 5(3), 287-299.

Sterne, J. (1995). World Wide Web marketing: integrating the Internet into your marketing strategy. John Wiley \& Sons, Inc.

Strauss, J. (2016). E-marketing. Routledge.

Taylor, M. J. y England, D. (2006). "Internet marketing: Web site navigation design issues". Marketing Intelligence \& Planning, 24(1), 77-85.

Tian, R. G. y Emery, C. (2002). "Cross-cultural issues in Internet marketing". Journal of American Academy of Business, Cambridge, 1(2), 217-224.

Wilson, R. F. y Pettijohn, J. B. (2008). "Search engine optimisation: A primer on outsourcing key tasks". Journal of Direct, Data and Digital Marketing Practice, 10(2), 133-149.

$\mathrm{Wu}$, S. (2002). "Internet marketing involvement and consumer behavior". Asia Pacific Journal of Marketing and Logistics, 14(4), 36-53.

Zineddine, M. (2016). "Los motores de búsqueda que se arrastran optimización de procesos: un enfoque de servidor web". Internet Research, 26 (1), 311-331.

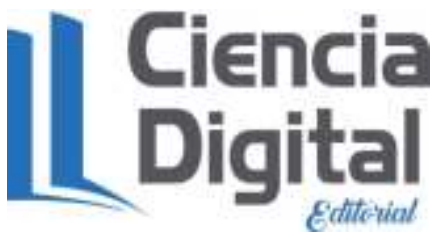


Para citar el artículo indexado.

Sánchez M., Fernández M. \& Mier-Terán J. • (2018). Principales características e implicaciones del marketing online. Revista electrónica Ciencia Digital 2(4), 78-92.

Recuperado desde: http://cienciadigital.org/revistacienciadigital2/index.php/CienciaDigital/article/view/211/18 6

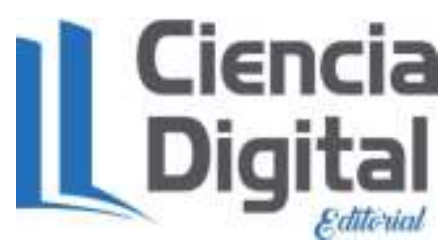

El artículo que se publica es de exclusiva responsabilidad de los autores y no necesariamente reflejan el pensamiento de la Revista Ciencia Digital.

El articulo queda en propiedad de la revista y, por tanto, su publicación parcial y/o total en otro medio tiene que ser autorizado por el director de la Revista Ciencia Digital.
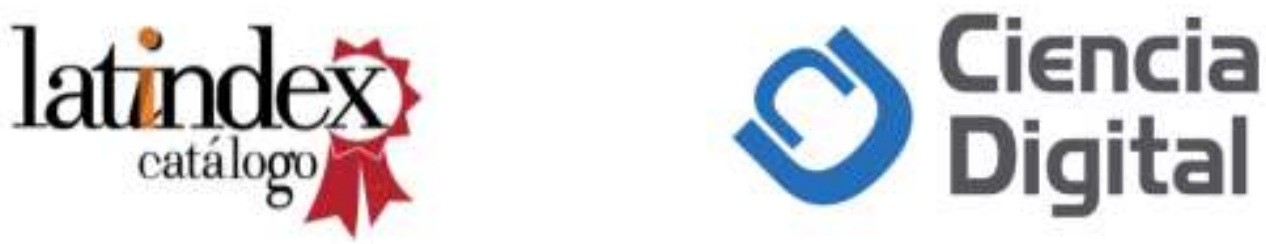